in many ways. Leaders felt that they got a valuable tool to handle different kinds of problematic situations at work. The model was also utilised within concerns about an organisational change.

Conclusions Mental first aid model was aimed to help work communities handle workplace incidents quickly on the spot. In addition to that, the model appeared to be more versatile instrument. It can be used to encourage discussing and solving challenging cases at work. It brings help easily available and strengthens work communities' own competence to deal with stressful situations. Inspired by positive experiences, a further use of the model is developed and more support persons are trained.

\section{NEED FOR NEW HUMAN FACTOR MODELS AND TOOLS IN THE SAFETY-CRITICAL NUCLEAR DOMAIN}

${ }^{1}$ Anna-Maria Teperi, ${ }^{1,2}$ Henriikka Ratilainen, ${ }^{1}$ Vuokko Puro. Finnish Institute of Occupational Health, Finland; ${ }^{2}$ Technical University of Tampere, Finland

\subsection{6/injuryprev-2016-042156.197}

Background In the nuclear industry and other safety-critical domains, recognising human behaviour as a key factor for improving safety culture is essential. Yet the focus has traditionally been on technical and procedural issues rather than human factors (HF). As HF remain both a resource and risk for nuclear safety, we need to improve our abilities to identify, analyse and learn about them. The aim of this study was to determine how the safety experts and supervisors of two nuclear power plants (NPPs) define HF, and to identify current HF procedures and the need for new HF tools as a part of safety management.

Methods We studied the current HF procedures in safety management using document analysis. Safety experts $(n=8)$ from two NPPs participated in a two-day workshop, in which a new HF tool was tested in the investigation of three operational events. We interviewed 22 safety experts and supervisors (20 from the NPPs, 2 from the regulator side), in order to study the current views and procedures of $\mathrm{HF}$, and the development needs for new HF tools in the domain.

Results Current safety procedures, for example, event analysis, still focus on technical aspects. HF procedures are seen as a way to inhibit individual errors. Several human performance tools were implemented at the NPPs, but none of them highlighted human success factors. Current HF tools were not actively used to analyse operational events, and no tools were used to summarise information from reports or their analyses for top management purposes. There was no model for normalising personnel's capacity after unwanted events at work: consequence management was seen more as the correcting of operative items.

Conclusions To improve HF management in the nuclear industry, practical HF tools are needed, as is stated in safety legislation and guidelines. To improve safety competence, it would be useful to further study the prerequisites and the hindrances of applying new HF tools in nuclear and other safety-critical industries.

\section{Strategies and Policies}

Parallel Mon 3.5

\section{POLICY MAKER'S PERCEPTIONS OF THE ROLE OF RESEARCH IN INJURY PREVENTION LEGISLATION}

${ }^{1}$ A Macpherson, ${ }^{1}$ L Rothman, ${ }^{2}$ Pamela Fuselli, ${ }^{3}$ Kathy Belton, ${ }^{4}$ Lise Olsen, ${ }^{5}$ lan Pike. ${ }^{1}$ Faculty of Health-School of Kinesiology and Health Science, York University, Toronto, Ontario, Canada; ${ }^{2}$ Parachute, Toronto, Ontario, Canada; ${ }^{3}$ Injury Prevention Centre, Edmonton, Alberta, Canada; ${ }^{4}$ University of British Columbia, Okanagan, Kelowna, British Columbia, Canada; ${ }^{5}$ Department of Paediatrics, University of British Columbia, BC Injury Research and Prevention Unit, Child and Family Research Institute, Vancouver, British Columbia, Canada

\subsection{6/injuryprev-2016-042156.198}

Background Injury prevention policy is crucial for the safety of Canada's children; however, legislation is not adopted uniformly across the country. Researchers and policy makers must work together to develop effective legislation that is evidence-based but little is known regarding policy makers views regarding the importance of research in facilitating the legislative process.

Methods Purposive snowball sampling identified individuals involved in injury prevention practice and policy throughout Canada. In an online survey, respondents identified injury topics relevant to them and rated the importance of enablers to injury legislation using a 5 point Likert scale.

Results There were 57 respondents with representation from all 10 provinces. The most common topics were, bicycle helmets (77\%), cell phone-distracted driving (63\%), booster seats (49\%), ski helmets (42\%), and graduated driver's licensing (37\%). The most frequently identified enabler was that research/surveillance was readily available (59\%). Other commonly reported research enablers were: research of sufficient quality/quantity that was easy to understand and in a useful format and affiliation of researchers with reputable organisations. Less important was researchers having similar priorities as policy makers and understanding the policy process. The importance of different research enablers varied by injury topic.

Conclusions Although policy makers identified the importance that injury prevention research was readily available, it appeared to be less important that researchers had similar priorities or understood the policy process, with variability by topic. This presents a challenge for researchers to conduct timely research and emphasises the need for ongoing relationships with policy makers with discussions early in the research process. This would facilitate the development of common injury prevention priorities to ensure research is used effectively in the legislative process.

\section{TARGET PROGRAMME FOR THE PREVENTION OF HOME AND LEISURE INJURIES 2014-2020}

Kari Paaso, Pirjo Lillsunde. Ministry of Social Affairs and Health, Helsinki

\subsection{6/injuryprev-2016-042156.199}

Background Almost 90 per cent of the deaths caused by accidents and more than 70 per cent of the accidents causing an injury occur at home and in leisure time. The most common type of accident is falling and tumbling. One third of the fatal accidents occur under the influence of alcohol.

Description of the problem The treatment of injuries and poisonings causes the second most inpatient periods in medical care and fourth most in institutional care within primary health care. 